\title{
La planificación del entrenamiento en el Fútbol amateur
}

\author{
Planning training in amateur soccer \\ Pablo Ariel Torres \\ Universidad Nacional de La Plata, Argentina \\ patunlp@gmail.com \\ iD https://orcid.org/0000-0002-2334-6114
}

\begin{abstract}
RESUMEN:
Este trabajo relevó, analizó y caracterizó la planificación de los entrenamientos de los clubes de fútbol masculino de la Liga Amateur Platense de la ciudad de La Plata (Buenos Aires, Argentina), entre los años 2013-2019. El poco conocimiento científico alrededor del entrenamiento del fútbol en este contexto específico es un inconveniente para la organización del proceso de entrenamiento, lo que lleva muchas veces, adaptar el modelo del alto rendimiento a un ámbito totalmente diferente y con menores recursos. Ante este desafío optamos apelar el problema de investigación desde la técnica de investigación cuantitativa y descriptiva, específica para la recolección de datos. Se diseñó un cuestionario de elaboración propia para ser aplicado mediante entrevista personal a los preparadores físicos del primer equipo de varios clubes platenses de fútbol masculino amateur. Mediante estos cuestionarios estructurados, estandarizados y cerrados, se recolectó información que nos permitió concluir que: la planificación en los clubes tiende a ser vulnerable, variable y consecuentemente, improvisada, producto de los grandes inconvenientes y limitaciones del entorno amateur; la periodización, los métodos, los medios y las tareas de entrenamiento son acordes a la especialidad deportiva; las pretemporadas descritas fueron breves e insuficientes, para el óptimo desarrollo de la condición física de los jugadores; las etapas de transición no pudieron ser correctamente trabajadas, producto de las dificultades del entorno; el control de entrenamiento por parte de los preparadores físicos fue escaso; por último, la buena regularidad de los jugadores fue asociada al logro de puestos altos en la tabla de posiciones de los campeonatos.
\end{abstract}

Palabras clave: Fútbol, Entrenamiento, Planificación, Amateurismo.

\section{Abstract:}

This work revealed, analyzed and characterized the training planning of the men's soccer clubs of the Platense Amateur League of the city of La Plata, Buenos Aires, Argentina, between 2013 and 2019. The little scientific knowledge about soccer training in this specific context is an inconvenience for the organization of the training process, which often leads to adapting the high performance model to a totally different environment with fewer resources. Faced with this challenge, we opted to address the research problem from a quantitative and descriptive research technique, specific for data collection. A self-made questionnaire was designed to be applied in personal interviews to the physical trainers of the first team of several amateur men's soccer clubs in La Plata. Through these structured, standardized and closed questionnaires, information was collected that allowed us to conclude the following: club planning tends to be vulnerable, variable and consequently improvised, as a result of the great inconveniences and limitations of the amateur environment; the periodization, the methods, the means and the training tasks are in accordance with the sports specialty; the pre-seasons described were short and insufficient, for the optimal development of the physical condition of the players; the transition stages could not be worked out correctly as a result of the difficulties of the environment; training control by physical trainers was poor; and, finally, the good consistency of the players was associated with the achievement of high positions in the championship standings.

KEYWORDS: Football, Training, Planning, Amateurism.

\section{INTRODUCCIÓN}

El proceso de entrenamiento puede ser definido a partir de tres palabras claves: planificar, intervenir $y$ evaluar (Castellano \& Casamichana, 2016). Siguiendo esta definición, aparece como vital el rol de la 
planificación en el entrenamiento deportivo. Ésta se realiza para elaborar y establecer un plan guiado por objetivos específicos, apoyándose sobre leyes, principios y reglas, determinadas por la finalidad deportiva y edades de los deportistas (García, Navarro \& Ruiz, 1996). Además, proyecta ciertos medios, métodos y tareas para el ámbito de actuación. García et al. (1996) discriminan a los siguientes eslabones como componentes de una planificación: estudios previos (diagnóstico de las condiciones y del contexto); definición de objetivos; calendario de competición; racionalización de las estructuras intermedias (periodizaciones del entrenamiento); elección de los medios y métodos de trabajo; distribución de las cargas de entrenamiento (dosificación del entrenamiento). Finalmente, creemos importante sumarle el componente evaluativo o del control del entrenamiento (Martínez Poch, SF).

En la bibliografía, se clasifica a los modelos de planificación en dos grandes grupos: tradicionales y contemporáneos (Camacho, Ochoa \& Rincón, 2019), entre los primeros encontramos, el clásico de Matveev, el de Macrociclo "ATR", y el de Verkhoshansky. Estos modelos tradicionales son eficaces en la mejora del rendimiento de los deportistas y también, han ido variando con el avance de los años (González Ravé, Navarro, Valdivielso \& Pereira Gaspar, 2015). Además, puede decirse que fueron diagramados en función de los deportes individuales, siendo insuficientes e inespecíficos para los deportes de conjunto, aunque con diversas variaciones han sido aplicados en ellos. Los nuevos sistemas de entrenamientos "integrales" o "contemporáneos" son específicos de cada disciplina deportiva, ya que perciben y piensan al deporte seleccionado (y al deportista) desde el enfoque de la complejidad. Los modelos contemporáneos tienen como pilar, el uso de situaciones reales de juego en la programación de las tareas de entrenamiento (Vera Rivera \& Merchán Osorio, 2011; Martínez Poch, SF; Tassi, 2017a). Estas maneras de pensar la preparación deportiva son consideradas globales, ya que trabajan los componentes del entrenamiento de manera conjunta (técnico, táctico, condicional y psicológico). Entre ellos se encuentran el Entrenamiento Estructurado (Seirulo.lo Vargas, 2017) y la Periodización Táctica (Tamarit Gimeno, 2013), ambas coinciden en que las estructuras que conforman la preparación de los deportistas, deben ser trabajadas de forma integrada y simultánea en el campo (Arjol, 2012; Tassi, 2017a). Los "nuevos" modelos son apoyados con una fuerte estructura tecnológica para la cuantificación de la carga y control del entrenamiento, además de estar pensada y dirigida para el alto rendimiento (Camacho et al., 2019). La elección por algunos de estos modelos debe ser específica al deporte a entrenar y a los deportistas, tanto por su experiencia y nivel, ya que son considerados condicionantes de la planificación (Navarro, 2001).

En este estudio abordamos la planificación de los entrenamientos en el fútbol (amateur). Éste es un deporte de equipo, foco de numerosas investigaciones que intentan describir el desarrollo de la condición física en los entrenamientos y la planificación de los mismos. Entre la abundante bibliografía sobre esta temática, sobresale el artículo de Moliner, Legaz, Munguía \& Medina (2010), quienes investigaron las características de planificación del entrenamiento condicional de diversos deportes de equipo españoles, incluido el fútbol de élite. Este cúmulo bibliográfico sobre la planificación deportiva no se traslada al deporte amateur. El poco conocimiento científico alrededor del entrenamiento del fútbol en este contexto específico (Búa \& García, 2019), es un inconveniente para la organización del proceso de entrenamiento, lo que lleva muchas veces, adaptar el modelo del alto rendimiento a un contexto totalmente diferente y con menores recursos.

Entre las pocas investigaciones halladas, que aportaron datos sobre el entrenamiento del fútbol amateur, destacamos la mención que realiza Bangsbo (1997) al describir la organización de los entrenamientos. Su trabajo está orientado tanto para elite como para los "aficionados", aportando que éstos deben centrarse en el entrenamiento aeróbico y anaeróbico de la velocidad. En este sentido, Naclerio (2011) agrega que los objetivos de los entrenamientos suelen buscar la mejora en: la fuerza, la resistencia aeróbica y la capacidad de repetir sprints. Otros autores orientaron sus investigaciones al establecimiento de perfiles físicos y morfológicos de los jugadores amateurs, y también, a la comparación con jugadores semi-profesionales (Búa, Rodríguez \& García, 2013; Búa \& García, 2019; Azcárate, Los Arcos \& Yanci, 2016; Yanci \& Los Arcos, 2015). En relación a la planificación de los entrenamientos del fútbol no-profesional, García Morato (2018) 
elaboró un trabajo académico sobre la organización de un ciclo de entrenamiento para el fútbol federado amateur, en cual describió contenidos, tareas, organización y control de los entrenamientos.

Este trabajo, parte de la Tesina final de la Licenciatura en Educación Física (Universidad Nacional de La Plata): 'La preparación fisica en la Liga Amateur Platense de fútbol", tiene como objetivo relevar y analizar las características de la planificación del entrenamiento de los clubes de fútbol masculino de la Liga Amateur Platense (LAP) de la ciudad de La Plata (Buenos Aires, Argentina), específicamente entre los años 2013-2019. Esta liga se encuentra afiliada indirectamente a la Asociación del Fútbol Argentino y está compuesta por 30 clubes (Liga Amateur Platense de Fútbol, 2015; El Día, 2013) los cuales tienen en sus líneas a gran cantidad de jugadores de la región. Estos futbolistas "aficionados" (no son profesionales), compiten una vez por semana y tienen dos o tres estímulos de entrenamiento cada siete días, coincidiendo con las características que describen diferentes autores para definir a los jugadores amateurs (Azcárate et al., 2016; Van der Horst, Wouter Smits, Petersen, Goedhart, \& Backx, 2015; Krist, Beijsterveldt, Backx \& De Wit, 2013).

El escaso tratamiento académico sobre el fútbol amateur platense nos presenta una oportunidad para aportar un antecedente de estudio, con el que se pretende aportar datos y análisis de las características de la planificación de los entrenamientos de varios clubes, otorgando al campo de la Educación Física, nociones actualizadas que puedan utilizarse en futuras investigaciones.

\section{Material Y MÉTODOS}

partir de los objetivos, se diseñó un cuestionario de elaboración propia para ser aplicado mediante entrevista personal a los preparadores físicos (PF) del primer equipo de varios clubes de fútbol masculino de la LAP, que estuvieron en sus cargos entre los años 2013-2019. Este trabajo de campo se llevó a cabo entre los meses de febrero de 2018 y abril de 2019. Ante este desafío optamos apelar el problema de investigación desde la técnica de investigación cuantitativa y descriptiva, específica para la recolección de datos (Marradi, Archenti \& Piovanni, 2007). Mediante los cuestionarios estructurados, estandarizados y cerrados, se recolectó información que nos permitió conocer sobre la planificación de los entrenamientos en el mundo del fútbol amateur. Estos datos fueron extraídos a través del uso de unas planillas, con preguntas y un abanico de opciones preseleccionadas para ser respondidas por los sujetos consultados. La forma de registrar la información fue mediante lapicera y papel impreso con las guías de consignas. El protocolo del cuestionario aplicado sistemáticamente, nos permitió contrastar y comparar los datos obtenidos de manera directa entre cada aplicación a los diferentes PF. La elección de este instrumento se justificó porque su procedimiento y los resultados arrojados, son de fácil aplicación y comprensión.

Se solicitó la colaboración de los profesionales contactándolos directamente mediante llamada telefónica y vía mail. Se les explicó las características del trabajo a realizar y luego de su aceptación, se establecieron fechas para administrar el cuestionario mediante entrevista personal. Finalmente fueron entrevistados $10 \mathrm{PF}$, quedando otros afuera del estudio por la imposibilidad de un encuentro. Con el cuestionario se estudiaron variables de los siguientes bloques temáticos:

- Las relacionadas con las características del cuerpo técnico. En este bloque de preguntas se recogió información acerca del número de personas que componen el cuerpo técnico, sobre la formación deportiva y académica, experiencia y antigüedad del PF.

- Las emparentadas con la información de la planificación realizada durante la temporada: momento de inicio de la pretemporada, modelos de planificación empleados (indicando si seguían el modelo periodización de pretemporada, competición y transición), proposición de objetivos previos al inicio de temporada, volumen de horas semanales, cantidad de sesiones y duración según el periodo, tipo, modelo y metodología de planificación empleado, manera de realización del trabajo condicional 
en las sesiones de entrenamiento y porcentaje de tiempo dedicado a las capacidades físicas según momento de la temporada.

- Las que se asocian a los medios y las herramientas de entrenamiento empleadas: los materiales e instalaciones de entrenamiento disponibles (la cantidad, la frecuencia de utilización, la disponibilidad e institución o persona a la que pertenecen) y los medios de entrenamiento elegidos según el momento de la temporada.

- Las relacionadas a las capacidades condicionales: objetivos del entrenamiento de la fuerza y tareas para su entrenamiento, herramientas utilizadas en la prevención de lesiones, métodos para trabajar la resistencia y la flexibilidad.

- Las de control del entrenamiento: forma de controlar, verificar los entrenamientos/competiciones (estadística, test, vídeo, etc.), material empleado, frecuencia, momento y periodo de temporada en que se realiza.

- Las de información general. Se consultó sobre la regularidad de los jugadores a entrenamientos y partidos, puestos generales en la temporada y sobre la suspensión de los entrenamientos.

Por último, la sistematización de los datos obtenidos en el trabajo de campo se realizó mediante el análisis estadístico, a partir del software SPSS versión 19.0 para Windows. Se calcularon estadísticos descriptivos y asociaciones entre las variables estudiadas. En el análisis se consideraron significativas aquellas diferencias que tuvieran una probabilidad de error inferior al $5 \%(\mathrm{p}<0.05)$.

\section{Resultados}

A continuación, se muestran los principales datos que arrojaron los cuestionarios a los $10 \mathrm{PF}$, los cuales fueron seleccionados y resumidos en una matriz de datos, creada específicamente para relevar toda la información brindada por los profesores.

\section{Características de los cuerpos técnicos.}

- Solamente el $60 \%$ de los PF estuvo a cargo de la preparación física durante una temporada completa (un año).

- La composición de los cuerpos técnicos, en cuanto a cantidad, fue de tres a ocho integrantes.

- El 70\% afirmó estar informado sobre el planteamiento de objetivos institucionales (en conjunto: dirigentes, directores técnicos y $\mathrm{PF}$, entre otros).

- El 70\% de los PF realizó su trabajo de manera individual, el resto de forma conjunta con otro colega.

- El 90\% de los PF entrevistados cuenta con un título (Profesor en Educación Física) proveniente de la Universidad Nacional de La Plata, el restante de un Instituto de Educación Física. También, el 90\% afirmó continuar capacitándose en temas relacionados a la preparación física.

- $\quad$ El 80\% de los PF han sido jugadores de la LAP.

- La antigüedad de los entrevistados, como PF, va desde la nula experiencia a los 20 años de actividad.

\section{Características de los modelos de planificación y periodización del entrenamiento en el fútbol amateur.}

- La mitad de los PF programó actividades para la etapa transición. El resto no pudo planificar esta etapa por no poder completar la temporada trabajando. 
- En general, la resistencia aeróbica, la fuerza y la flexibilidad fueron los contenidos a trabajar durante la etapa de transición. Este entrenamiento programado, lo debían realizar los jugadores por su propia cuenta, con una frecuencia de tres veces por semana y una duración de 60 minutos aproximadamente.

- Los nueve PF que llevaron a cabo alguna pretemporada, la realizaron durante cuatro y seis semanas de preparación.

- En general, el volumen de horas de entrenamiento semanal, fue mayor durante la pretemporada. Aunque también varía la cantidad de horas de entrenamiento (pretemporada, competición y transición) en cada caso.

- Los entrevistados planificaron tanto a partir de la metodología integral como la clásica.

- Todos afirmaron que a la hora de planificar tuvo incidencia tanto la evidencia científica como la experiencia en el trabajo.

\section{Contenidos, métodos, medios, tareas y control del entrenamiento.}

En relación a las capacidades condicionales:

- Todos los casos llevaron a cabo ejercicios específicos para la prevención de lesiones, orientación del entrenamiento de la fuerza mayormente utilizada por los PF (en comparación con el incremento del rendimiento). Entre las tareas utilizadas por los PF para el entrenamiento de la fuerza se encuentran los ejercicios con el propio peso corporal, el trabajo de musculación a partir de pesas (mayormente utilizada), ejercicios olímpicos, la utilización de bandas y medicine ball (menos utilizada).

- Los métodos favoritos de los PF, para el entrenamiento de la resistencia específica, fueron el intermitente de alta intensidad en pretemporada y el intermitente de sprints para la competencia. Además, utilizaron para entrenar esta capacidad: los métodos continuos, fraccionados y los integrales (conjuntamente con la técnica y la táctica) entre otras opciones.

- Para el abordaje de la flexibilidad, los PF usaron preferentemente a la elongación pasiva, y también, a los ejercicios de movilidad articular y elongación activa.

En cuanto a los medios de entrenamientos, el control de los estímulos y la ubicación de los contenidos de la sesión, los PF describieron a los siguientes:

- Medios: circuitos de resistencia, técnicos- tácticos y de fuerza, RSA, multi-saltos, carreras resistidas, formación teórica, juegos modificados, fútbol informal y juegos reducidos (además, de los ya nombrados).

- El 50\% realizó test (evaluaciones físicas).

- Algunos (el 30\%) afirmaron trabajar la parte "física" previa al trabajo técnico/táctico, otro (10\%) posteriormente y los restantes $(60 \%)$ sin una ubicación específica en la sesión de entrenamiento.

A continuación en la Tabla 1 , se resumen los datos relacionados a los contenidos de entrenamiento durante la pretemporada y la etapa de competencia. 
TABLA 1

Porcentajes de tiempo aproximados dedicados a cada contenido de entrenamiento. Preparación física- táctica/técnica. Trabajo condicional

\begin{tabular}{|c|c|c|c|c|c|c|}
\hline Caso & & Pretemporada & Competencia & Capacidad física & Pretemporada & Competencia \\
\hline 1 & $\begin{array}{l}\text { Trabajo } \\
\text { técnico/ } \\
\text { táctico \% } \\
\text { Preparación } \\
\text { fúsica \% }\end{array}$ & 100 & 50 & $\begin{array}{l}\text { Resistencia } \\
\text { Fuerza } \\
\text { Prevención de lesiones } \\
\text { Flexibilidad }\end{array}$ & $\begin{array}{l}50 \\
30 \\
10 \\
10\end{array}$ & $\begin{array}{l}50 \\
30 \\
10 \\
10\end{array}$ \\
\hline 2 & $\begin{array}{l}\text { Trabajo } \\
\text { técnico/ } \\
\text { táctico \% } \\
\text { Preparación } \\
\text { física \% }\end{array}$ & 30 & 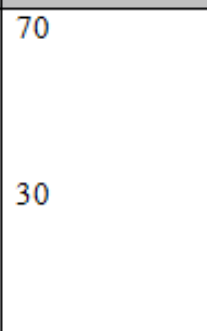 & $\begin{array}{l}\text { Resistencia } \\
\text { Fuerza } \\
\text { Prevención de lesiones/ } \\
\text { Flexibilidad } \\
\text { Velocidad }\end{array}$ & $\begin{array}{l}70 \\
15 \\
10 \\
5\end{array}$ & $\begin{array}{l}45 \\
20 \\
30 \\
5\end{array}$ \\
\hline 3 & $\begin{array}{l}\text { Trabajo } \\
\text { técnico/ } \\
\text { táctico \% } \\
\text { Preparación } \\
\text { física \% }\end{array}$ & 35 & 65 & $\begin{array}{l}\text { Resistencia } \\
\text { Fuerza/Prevención de } \\
\text { lesiones } \\
\text { Flexibilidad } \\
\text { Velocidad/coordinación }\end{array}$ & $\begin{array}{l}50 \\
30 \\
- \\
20\end{array}$ & $\begin{array}{l}30 \\
30 \\
- \\
40\end{array}$ \\
\hline 4 & $\begin{array}{l}\text { Trabajo } \\
\text { técnico/ } \\
\text { táctico \% } \\
\text { Preparación } \\
\text { física \% }\end{array}$ & 40 & 30 & $\begin{array}{l}\text { Resistencia } \\
\text { Fuerza/Prevención de } \\
\text { lesiones } \\
\text { Flexibilidad }\end{array}$ & $\begin{array}{l}60 \\
35 \\
5\end{array}$ & $\begin{array}{l}50 \\
45 \\
5\end{array}$ \\
\hline 5 & $\begin{array}{l}\text { Trabajo } \\
\text { técnico/ } \\
\text { táctico \% } \\
\text { Preparación } \\
\text { física \% }\end{array}$ & 40 & 50 & $\begin{array}{l}\text { Resistencia } \\
\text { Fuerza } \\
\text { Prevención de lesiones } \\
\text { Flexibilidad }\end{array}$ & $\begin{array}{l}70 \\
10 \\
10 \\
10\end{array}$ & $\begin{array}{l}70 \\
10 \\
10 \\
10\end{array}$ \\
\hline 6 & $\begin{array}{l}\text { Trabajo } \\
\text { técnico/ } \\
\text { táctico \% } \\
\text { Preparación } \\
\text { física \% }\end{array}$ & 60 & 30 & $\begin{array}{l}\text { Resistencia } \\
\text { Fuerza } \\
\text { Prevención de lesiones } \\
\text { Flexibilidad }\end{array}$ & $\begin{array}{l}45 \\
45 \\
5 \\
5\end{array}$ & $\begin{array}{l}40 \\
30 \\
15 \\
15\end{array}$ \\
\hline
\end{tabular}




\begin{tabular}{|c|c|c|c|c|c|c|}
\hline 7 & $\begin{array}{l}\text { Trabajo } \\
\text { técnico/ } \\
\text { táctico \% } \\
\text { Preparación } \\
\text { física \% }\end{array}$ & - & 65 & $\begin{array}{l}\text { Resistencia } \\
\text { Fuerza } \\
\text { Prevención de lesiones } \\
\text { Flexibilidad }\end{array}$ & $\begin{array}{l}30 \\
50 \\
10 \\
10\end{array}$ & $\begin{array}{l}40 \\
20 \\
20 \\
20\end{array}$ \\
\hline 8 & $\begin{array}{l}\text { Trabajo } \\
\text { técnico/ } \\
\text { táctico \% } \\
\text { Preparación } \\
\text { física \% }\end{array}$ & $\begin{array}{l}50 \\
50\end{array}$ & 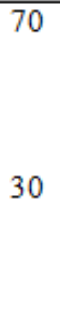 & $\begin{array}{l}\text { Resistencia } \\
\text { Fuerza } \\
\text { Prevención de lesiones } \\
\text { Flexibilidad } \\
\text { Otro: agilidad }\end{array}$ & $\begin{array}{l}30 \\
30 \\
35 \\
- \\
5\end{array}$ & $\begin{array}{l}30 \\
30 \\
35 \\
- \\
5\end{array}$ \\
\hline 9 & $\begin{array}{l}\text { Trabajo } \\
\text { técnico/ } \\
\text { táctico \% } \\
\text { Preparación } \\
\text { física \% }\end{array}$ & 75 & $\begin{array}{l}60 \\
40\end{array}$ & $\begin{array}{l}\text { Resistencia } \\
\text { Fuerza } \\
\text { Prevención de lesiones } \\
\text { Flexibilidad } \\
\text { Otro: agilidad }\end{array}$ & $\begin{array}{l}55 \\
20 \\
10 \\
10 \\
5\end{array}$ & $\begin{array}{l}50 \\
25 \\
10 \\
10 \\
5\end{array}$ \\
\hline 10 & $\begin{array}{l}\text { Trabajo } \\
\text { técnico/ } \\
\text { táctico \% } \\
\text { Preparación } \\
\text { física \% }\end{array}$ & - & 65 & $\begin{array}{l}\text { Resistencia } \\
\text { Fuerza } \\
\text { Prevención de lesiones } \\
\text { Flexibilidad } \\
\text { Otro: velocidad- } \\
\text { agilidad-coordinación }\end{array}$ & & $\begin{array}{l}50 \\
10 \\
20 \\
10 \\
10\end{array}$ \\
\hline
\end{tabular}

Fuente: elaboración propia.

A la izquierda: dedicación del tiempo (en porcentajes) de entrenamiento a la preparación física y a la técnica/ táctica, según periodo de entrenamiento (pretemporada y competencia). A la derecha: dedicación del tiempo de entrenamiento a cada capacidad física, según periodo de la temporada.

En general, podemos identificar que los cuerpos técnicos, hicieron hincapié en el trabajo de la condición física de los jugadores durante las pretemporadas y al entrenamiento técnico-táctico, durante la competencia. En cuanto al uso que hizo cada profesor de los tiempos de entrenamiento, podemos decir que fue variable, ya que algunos priorizaron ciertas capacidades con mayor énfasis y otros optaron por diferentes estrategias durante las prácticas.

\section{Las condiciones espaciales, materiales y problemáticas de los clubes de la LAP}

Los 30 clubes que componen la liga tienen características sociales, económicas, materiales y espaciales, muy distintas. En la Tabla 2, se muestran los datos hallados en relación al espacio, materiales, asistencia de los jugadores (a partidos y entrenamientos) y posición en la tabla, de cada caso entrevistado. 
TABLA 2

Posición en la tabla, regularidad, materiales y espacios por caso.

\begin{tabular}{|c|c|c|c|c|}
\hline Caso & Posición en la tabla & $\begin{array}{l}\text { Regularidad de los } \\
\text { jugadores }\end{array}$ & $\begin{array}{l}\text { Materiales para el } \\
\text { entrenamiento de la } \\
\text { fuerza }\end{array}$ & Espacios \\
\hline 1 & Ultimos puestos & Mala & Sin materiales. & $\begin{array}{l}\text { Una cancha y un } \\
\text { espacio reducido } \\
\text { techado }\end{array}$ \\
\hline 2 & Primeros puestos & Buena & $\begin{array}{l}\text { Pesas, barras, } \\
\text { mancuemas }\end{array}$ & $\begin{array}{l}\text { Dos canchas } \\
\text { disponibles y un } \\
\text { espacio techado. }\end{array}$ \\
\hline 3 & Primeros puestos & Muy buena & $\begin{array}{l}\text { Bandas elásticas, } \\
\text { medicine ball, Pesas, } \\
\text { barras, mancuemas }\end{array}$ & Una cancha. \\
\hline 4 & Puestos medios & Buena & $\begin{array}{l}\text { Pesas, barras, } \\
\text { mancuemas }\end{array}$ & $\begin{array}{l}\text { Una cancha, } \\
\text { alquiler de sintética } \\
\text { en caso de lluvia }\end{array}$ \\
\hline 5 & Primeros puestos & Muy buena & $\begin{array}{l}\text { Pesas, barras, } \\
\text { mancuemas }\end{array}$ & $\begin{array}{l}\text { Tres canchas y un } \\
\text { espacio techado } \\
\text { reducido. }\end{array}$ \\
\hline 6 & Puestos medios & Buena & $\begin{array}{l}\text { Pesas, barras, } \\
\text { mancuemas }\end{array}$ & $\begin{array}{l}\text { Una cancha y un } \\
\text { espacio techado } \\
\text { (sede del club). }\end{array}$ \\
\hline 7 & Primeros puestos & Buena & $\begin{array}{l}\text { Pesas, barras, } \\
\text { mancuemas (propiedad } \\
\text { de los PF) }\end{array}$ & $\begin{array}{l}\text { Una cancha y un } \\
\text { espacio techado } \\
\text { reducido. }\end{array}$ \\
\hline 8 & Primeros puestos & Muy buena & $\begin{array}{l}\text { Pesas, barras, } \\
\text { mancuemas }\end{array}$ & $\begin{array}{l}\text { Tres canchas, un } \\
\text { espacio techado y } \\
\text { sede. }\end{array}$ \\
\hline 9 & Ultimos puestos & Buena & $\begin{array}{l}\text { Pesas, barras, } \\
\text { mancuemas }\end{array}$ & $\begin{array}{l}\text { Una cancha y } \\
\text { espacio techado } \\
\text { reducido }\end{array}$ \\
\hline 10 & Ultimos puestos & Mala & Sin materiales. & $\begin{array}{l}\text { Una cancha para } \\
\text { entrenar(alquilada) }\end{array}$ \\
\hline
\end{tabular}

Fuente: elaboración propia.

*Todos los casos utilizarán los parques y plazas de la ciudad para entrenar (por falta de disponibilidad de espacios en el propio club o cuidar los terrenos de juego). 
En cuanto a las dificultades de la planificación en los clubes, los PF señalaron a la asistencia y al compromiso por parte de los jugadores, como principales inconvenientes, y en segundo lugar, a las suspensiones ocasionales de entrenamientos y partidos por cuestiones climáticas (relacionado al vulnerable estado de los campos de juego):

El 30\% afirmó que los entrenamientos se suspenden por clima lluvioso.

La regularidad de los jugadores a las prácticas, según los PF fue: mala (según el 20\%), buena (el 50\%) y muy buena (el 30\%). Aclarando que "muy buena" refiere a mínimas ausencias durante la temporada.

A esta última variable (Regularidad de los jugadores a entrenamientos) pudimos relacionarla con: posición en la tabla del campeonato. En la siguiente Tabla 3, se resume la correlación:

TABLA 3

Correlación de Rho de Spearman entre las variables posición en la tabla del campeonato y regularidad de los jugadores.

\begin{tabular}{|c|c|c|c|c|}
\hline & $\begin{array}{l}\text { Correlación entre } \\
\text { las variables } \\
\text { posición en la tabla } \\
\text { del campeonatoy } \\
\text { regularidad delos } \\
\text { jugadores }\end{array}$ & & $\begin{array}{l}\text { Posición en la tabla } \\
\text { del campeonato }\end{array}$ & $\begin{array}{l}\text { Regularidad de los } \\
\text { jugadores a los } \\
\text { entrenamientos }\end{array}$ \\
\hline \multirow[t]{6}{*}{ Rho de Spearman } & \multirow[t]{3}{*}{$\begin{array}{l}\text { Posición en la tabla } \\
\text { del campeonato }\end{array}$} & $\begin{array}{l}\text { Coeficiente de } \\
\text { correlación }\end{array}$ & 1 &, $779(* *)$ \\
\hline & & Sig. (bilateral) & & 0,01 \\
\hline & & $\mathrm{N}$ & 10 & 10 \\
\hline & \multirow{3}{*}{$\begin{array}{l}\text { Regularidad de los } \\
\text { jugadores a los } \\
\text { entrenamientos }\end{array}$} & $\begin{array}{l}\text { Coeficiente de } \\
\text { correlación }\end{array}$ &, $779(* *)$ & 1 \\
\hline & & Sig. (bilateral) & 0,01 & . \\
\hline & & $\mathrm{N}$ & 10 & 10 \\
\hline
\end{tabular}

Fuente: elaboración propia.

${ }^{*}$ La correlación es significativa al nivel 0,01 (bilateral).

La variable posición en la tabla del campeonato, correlacionó positivamente con la regularidad de los jugadores a los entrenamientos, por lo que tener una mejor o peor posición en el torneo, tendió a coincidir con una mejor o peor regularidad de los jugadores a entrenamientos y partidos, respectivamente.

\section{Discusión}

\section{Características de los cuerpos técnicos.}

Los PF de LAP planifican teniendo en cuenta su experiencia en el ámbito de la preparación física y la evidencia científica recuperada de bibliografía sobre el entrenamiento del fútbol (de índole profesional). Según los hallazgos mencionados en el apartado anterior, no todos los PF tuvieron la oportunidad de trabajar una temporada completa y no todos admitieron estar al tanto de los propósitos institucionales de cada club. 
Varios autores sostienen que una adecuada planificación a nivel institucional y luego del cuerpo técnico, es pilar fundamental para trazar objetivos, ya que se trata de un proceso continuo que permite comparar resultados previstos y alcanzados (García et al., 1996; Martínez Poch, SF).

\section{Características de los modelos de planificación y periodización del entrenamiento en el fútbol amateur.}

La forma de programar en el contexto amateur investigado no se corresponde a lo que entendemos por proceso de entrenamiento: planificar, intervenir y evaluar (Castellano \& Casamichana, 2016). Los datos muestran que la planificación es muy vulnerable por: los cambios constantes que presenta el amateurismo, la escasez de recursos, la suspensión de los entrenamientos y la inasistencia de los jugadores. Además, la evaluación es utilizada para controlar y programar los entrenamientos solamente en un $50 \%$ de los casos.

$\mathrm{Al}$ igual que lo analizado en la bibliografía sobre el fútbol profesional (Lapresa Ajamil, Arana Idiakez, Carazo Gómez \& Ponce de León Elizondo (2002), casi todos los PF encuestados dividen la temporada en preparatoria, competitiva y transición. Según datos obtenidos, en la mayoría de los casos la pretemporada rondaba las cuatro y seis semanas de preparación previa a la competencia. Estos mismos autores (Lapresa Ajamil et al., 2002) dicen que se requieren de seis a ocho semanas de puesta a punto (aunque esto no se cumple en el profesionalismo argentino). Al realizarse la pretemporada en un periodo de cuatro semanas, los jugadores podrían no alcanzar el nivel óptimo de rendimiento, por la cantidad de entrenamientos técnicos, tácticos y funcionales que se requieren para el fútbol. Una tendencia general de los PF fue que afirmaron trabajar la condición física de los jugadores, en mayor porcentaje de tiempo e intensidad durante la pretemporada. En cambio, la preparación técnica/táctica fue abordada con mayor énfasis, durante la competencia, cuando tomaba mayor relevancia el rol del Director Técnico. Sobre este tema, Bangsbo (1997) dice que no es conveniente que el periodo de pretemporada sea muy exigente, ya que esto incidiría en lesiones.

En cuanto al sistema de entrenamiento y de planificación utilizados, los PF utilizan tanto los modelos tradicionales como los integrales (Martínez Poch, SF; Tassi, 2017b). Aunque varios afirmaron elegir a estos últimos, no han seleccionado como prioridad, a los medios de entrenamiento característicos de ellos (juegos reducidos, rondos, etc.). En este sentido, Moliner et al. (2010) afirman que en los equipos españoles se utilizan ambos grupos de sistemas de planificación ("convencionales" e "integrales"). Los modelos teóricos "tradicionales", implican la consecución de picos de forma que no se adecuan a las demandas de los calendarios competitivos de los deportes de equipo, por lo que son limitados para el rendimiento en estas especialidades. Estos autores dicen también que es necesario la aplicación prioritaria de un trabajo integrado de los factores condicionales y técnico-tácticos, eligiendo como prioridad a los modelos "globales".

La mitad de los profesores no diagramaron tareas para la etapa de transición (entre fin de competición e inicio de pretemporada), por no haber continuado en sus cargos. Esto indica que en esos casos, no se cumplió con el objetivo propio de la etapa: la regeneración (Lapresa Ajamil et al., 2002) y tampoco se efectúa el principio de entrenamiento deportivo "reversibilidad" (Billat, 2002), el cual hace referencia al desentrenamiento que aparece de manera muy rápida al interrumpir el entrenamiento. Binkley, Sayers \& Saye (2008), aconsejan las tareas vacacionales de los jugadores, tanto para el alto rendimiento como también para el deporte amateur. Los cinco casos que dieron contenidos para la transición, prefirieron orientarlo a la resistencia aeróbica, la flexibilidad y la fuerza, en tres sesiones de entrenamiento de una hora de duración. Los autores que tomamos (Borbón \& Sánchez, 2013) priorizan el trabajo de la fuerza, lo que coincide con lo descrito por los PF de "La Liga" (aunque éstos también le suman el contenido aeróbico). Estos profesores describieron como "dificultoso", el cumplimiento del entrenamiento programado post competencia, por no poder ser monitoreados por ellos. 


\section{Contenidos, métodos, medios, tareas y control del entrenamiento.}

En cuanto a las principales cualidades a entrenar: Bangsbo (1997) aconseja para el nivel aficionado, hacer énfasis en la resistencia aeróbica y en la resistencia a la velocidad, coincidiendo con lo trabajado en los clubes, según los PF. Los datos recabados muestran que la resistencia específica de los futbolistas de LAP, se trabajaba mediante el método intermitente de alta intensidad en pretemporada y el intermitente de sprints para la competencia, ambos válidos según diferentes estudios (Borbón \& Sánchez, 2013; Casas, 2011). Para programar correctamente la metodología intermitente, es necesario evaluar la resistencia específica del fútbol y según la información recopilada, muy pocos profesores la han evaluado. Por otro lado, los métodos de entrenamiento integrales que permiten alcanzar intensidades altas a partir de ejercicios jugados con balón, también eran usados por los PF para el trabajo de la resistencia (Impellizzeri, 2007, cita en Borbón \& Sánchez, 2013). En relación al uso de estas tareas de entrenamiento, Dellal, Hill-Haas, Lago-Penas \& Chamari (2011) evidencian que en los juegos reducidos, los jugadores amateurs completan un menor porcentaje de pases exitosos, pierden mayor cantidad de posesión de balón y cubren menos distancia a máxima velocidad y perciben un esfuerzo mayor, en comparación a jugadores profesionales. Por lo que a la hora de planificar estas actividades integrales, se deberían tener en cuenta estas limitaciones, para conseguir los objetivos propuestos en este tipo de ejercicios. También varios afirmaron trabajar la velocidad a partir del RSA y las carreras de velocidad. Estos medios de entrenamiento para el trabajo de la resistencia específica del futbolista son válidos y correctamente elegidos (Casas, 2011; Spencer, Bishop, Dawson \& Goodman, 2005; Sánchez-Sánchez et al., 2016; Donskoy \& Zatsiorski 1988, cita en Naclerio, 2011).

En concordancia con Borbón \& Sánchez (2013), los PF eligen el trabajo en circuito para el abordaje de la fuerza orientado a la prevención de lesiones, porque permite entrenar en un espacio acotado, con muchos jugadores.

La flexibilidad es la capacidad menos trabajada según los PF, cuestión no favorable según los diferentes estudios (Timarán Benjumea \& Umbarila Quintana, 2016; Borbón \& Sánchez, 2013). En la Liga principalmente, la entrenan mediante ejercicios de elongación muscular pasiva.

En cuanto a la organización de los contenidos de entrenamiento en la sesión, la mayoría de los PF sostuvo no acordar una ubicación específica dentro de cada estímulo. Lo que concuerda con Moliner et al. (2010) quienes dicen que esta situación se debe generalmente, a que los PF no tienen responsabilidades en las tareas integrales/ tácticas, lo que merece una revisión en la manera de trabajar de los cuerpos técnicos.

\section{Las condiciones espaciales, materiales y problemáticas de los clubes de la LAP.}

Tras el armado de una matriz de datos, pudimos establecer una correlación positiva entre las variables: posicin en la tabla del campeonato y regularidad de los jugadores. Con la cautela que amerita el análisis estadístico con una población de $\mathrm{n}=10$, se ha mostrado una asociación entre la asistencia de los jugadores a los entrenamientos y los resultados deportivos. En este contexto deportivo amateur, parece suceder que quien logre mantener cierta estructura institucional, armonía grupal y entrenamientos periódicos, estables y completos, marcará la diferencia por sobre el resto, logrando puestos altos en los campeonatos. La suspensión ocasional de los entrenamientos por mal clima, sumado a los cambios constantes de espacios físicos de entrenamiento (la cancha del club, el parque, la plaza, la rambla, etc.), hace al trabajo de planificación complicado y poco predecible. Como estas situaciones no suceden en el alto rendimiento, tampoco son tenidas en cuenta en los estudios sobre este deporte, principal insumo bibliográfico de los PF. 


\section{Conclusiones}

Este trabajo simboliza una de las pocas investigaciones sobre las características de la planificación del entrenamiento en el fútbol amateur argentino. A continuación, resumimos las peculiaridades del trabajo en los clubes de LAP:

- La planificación tiende a ser vulnerable, variable y consecuentemente, improvisada. Esto es producto, principalmente, de los grandes inconvenientes y limitaciones del entorno amateur: una escasa bibliografía sobre planificación deportiva en estos contextos, la falta de continuidad de los cuerpos técnicos, las suspensiones ocasionales de partidos y los entrenamientos, la irregularidad de los jugadores, el déficit de materiales e instalaciones y la insuficiencia económica de los clubes, entre otras.

- En cuanto a los eslabones que componen una planificación modelo encontramos lo siguiente: la periodización, los métodos, los medios y las tareas de entrenamiento son acordes a la especialidad deportiva, según la bibliografía consultada. La etapa de pretemporada viene siendo breve, por lo que podría ser más extensa para asegurar una optimización de la condición física de los jugadores, aunque esto depende de la LAP. La etapa de transición y el control de entrenamiento merecen ser tomados con mayor importancia por los cuerpos técnicos, por su relevancia en el proceso de entrenamiento. Aclarando que la poca cantidad de evaluaciones y el "no" aprovechamiento de la etapa de transición son resultado, muchas veces, de los inconvenientes existentes en el amateurismo, ya nombrados más arriba.

- Los planteles con buena regularidad de sus jugadores a entrenamientos y partidos, lograron puestos altos en la tabla de posiciones de los campeonatos.

Finalizando, esta caracterización realizada sobre la planificación en los clubes de LAP, podría servir de reflexión y motivación para los actores del fútbol amateur, al momento de diagramar y evaluar los programas de entrenamiento. Además, se hace necesario orientar el trabajo investigativo hacia este masivo ámbito del entrenamiento deportivo, y de esta manera comenzar a producir datos científicos desde el amateurismo para no depender de los conocimientos producidos por y para el profesionalismo. Por ello, alentamos a construir un modelo de planificación específico que contenga eslabones, principios, métodos, periodizaciones, medios y tareas, y que considere las particularidades del fútbol amateur.

\section{REFERENCIAS}

Arjol, J. L. (2012). La planificación actual del entrenamiento en fútbol.: Análisis comparado del enfoque estructurado y la periodización táctica. Acción motriz, (8), 27-37. ISSN-e 1989-2837

Azcárate, U., Los Arcos, A., Yanci, J. (2016). Diferencias en la capacidad de aceleración, cambio de dirección y resistencia cardiovascular entre jugadores de fútbol amateur de diferentes categorías competitiva. Revista Universitaria de la Educación Física y el Deporte, (9). https://www.researchgate.net/publication/311571921

Bangsbo, J. (1997). El Entrenamiento de la Condición Física en el Futbol. Barcelona, España: Editorial Paidotribo.

Billat, V. (2002). Fisiología y Metodología del entrenamiento: de la teoría a la práctica. Barcelona, Epaña: Paidotribo.

Binkley, H., Sayers, A., \& Saye, B. E. (2008). Evaluación de la Aptitud Física para la Pretemporada de Jugadores de Fútbol de la Asociación Nacional de Deporte Universitario. PubliCE, 0 . https://g-se.com/evaluacion-de-la-aptitud-fisica-para-la-pretemporada-de-jugadores-de-futbol-de-la-asociac ion-nacional-de-deporte-universitario-1113-sa-P57cfb271bfe86

Borbón, O., \& Sánchez, F. (2013). Fútbol. Entrenamiento Actual de la Condición Física del Futbolista. MHSalud, $10(2), 1-131$. 
Búa, N., Rodríguez, A., García, G. (2013). «Perfil funcional y morfológico en jugadores de fútbol amateur de Mendoza, Argentina». Apunts: Medicina de l'esport, 48 (179), p. 89-96, https://www.raco.cat/index.php/Apunts/article /view/270165 [Consulta: 16-12-2020].

Bua, N., García, G. (2019).Comparación de la condición física entre futbolistas argentinos amateur y semi-profesional. Lecturas: Educación fisica y deportes, 24 (256). https://www.researchgate.net/publication/336533473

Camacho, J., Ochoa, N. y Rincón, N. (2019). Revisión teórica de la planificación tradicional y contemporánea en el entrenamiento deportivo. Revista digital: Actividad Fisica y Deporte, 5 (2): 171-181.

Casas, A. (2011). Entrenamiento de resistencia para deportes de conjunto. En F. Naclerio, Entrenamiento deportivo. Fundamentos y aportes en diferentes deportes. Madrid: Médica Panamericana editores

Castellano, J., \& Casamichana, D. (2016). El arte de planificar en fútbol. Barcelona: Fútbol De Libro.

Dellal, A., Hill-Haas, S., Lago-Penas, C., Chamari, K. (2011). Small-sided games in soccer: Amateur vs. professional players' physiological responses, physical and technical activities. The Journal of Strength \& Conditioning Research 25 (9), 2371-2381

El Día. (21 de abril de 2013). Cien años de historia. El Día. https://www.eldia.com/nota/2013-4-21-cien-anos-de-h istoria. Consultado el 1 de noviembre de 2019.

García J., Navarro, M. y Ruiz, J. (1996). Planificación del entrenamiento deportivo. Madrid: Gymnos Editorial Deportiva.

García Morato, D. (2018). Organización de un ciclo de entrenamiento para fútbol federado amateur. Recuperado de: $\underline{\mathrm{h}}$ ttps://www.researchgate.net/publication/325923460

González Ravé, F., Navarro, J., Valdivielso, J., Pereira Gaspar, P. (2015). La Planificación del Entrenamiento Deportivo: Cambios Vinculados a las Nuevas Formas de Entender las Estructuras Deportivas Contemporáneas. Revista de Entrenamiento Deportivo, 29 (1).

Krist, M., Beijsterveldt, A., Backx, F. \& De wit, G. (2013). Preventive exercises reduced injury-related costs among adult male amateur soccer players: A cluster-randomised trial. Journal of physiotherapy, 59. 15-23. https:// doi.org/10.1016/S1836-9553(13)70142-5.

Lapresa Ajamil, D., Arana Idiakez, J., Carazo Gómez, J., Ponce de León Elizondo, A. (2002). Orientaciones formativas para el entrenador de futbol. Universidad de La Rioja. Recuperado de : https://dialnet.unirioja.es/descarga/lib ro/379876.pdf

Liga Amateur Platense de fútbol. (14 de agosto de 2015). Historia. Organización de la federación. http://www.lapf.c om.ar/HistoriaConsulta.aspx Consultado el 1 de noviembre de 2019.

Marradi, A., Archenti, N., Piovanni, J.I. (2007). Metodologia de las Ciencias Sociales. Buenos Aires: Emecé.

Martínez Poch, G., (SF). Enciclopedia de entrenamiento del futbolista profesional. Cochabamba, Bolivia: Digital PrePress.

Moliner, U. D., Legaz, A., Munguía, I. D., \& Medina, R. E. (2010). Características de la planificación del entrenamiento en los deportes de equipo españoles de élite. Apunts. Educación Física y Deportes. (102), 62-69.

Naclerio, F. (2011). Entrenamiento deportivo. Fundamentos y aplicaciones en diferentes deportes. Madrid: Médica Panamericana editores.

Navarro, F. (2001). Modelos de planificación según el deportista y el deporte. Educación Física y Deporte, 9(67), 11-28.

Sánchez-Sánchez, J.; Hernández, C.; Marcos, V.; González, A.; Rodríguez, A., \& Carretero, M. (2016). Efecto de un entrenamiento intermitente con y sin cambios de dirección, sobre el rendimiento físico de jóvenes futbolistas. RETOS. Nuevas Tendencias en Educación Física, Deporte y Recreación, 30:70-75.

Spencer. M., Bishop, D., Dawson, B., Goodman, C. (2005). Physiological and metabolic responses of repeated-sprint activities : specific to field-based team sports. Sports Med, 35, 1025 - 1044

Seirulo.lo Vargas, F. (2017). El entrenamiento en los deportes de equipo. Barcelona: Biocorp Europa.

Tamarit Gimeno, X. (2013). Periodización Táctica vs Periodización Táctica. Vitor Frade Aclara. Madrid: MBF.

Tassi, J. (2017a). Metodologías y modelos de planificación en el fútbol actual: Acentuación psicológica en la periodización táctica y el microciclo estructurado. $12^{\circ}$ Congreso Argentino de Educación Física y Ciencias, 13 
al 17 de noviembre 2017, Ensenada, Argentina. Educación Física: construyendo nuevos espacios. EN: Actas (2017). Ensenada: Universidad Nacional de La Plata. Facultad de Humanidades y Ciencias de la Educación. Departamento de Educación Física. En Memoria Académica. Disponible en: http://www.memoria.fahce.unlp .edu.ar/trab eventos/ev.10204/ev.10204.pdf

Tassi, J. (2017b). El desarrollo psicológico en las tareas integradas en fútbol: Diferencias entre situaciones de juego reducidas y globales. 12 Congreso Argentino de Educación Física y Ciencias, 13 al 17 de noviembre 2017, Ensenada, Argentina. Educación Física: construyendo nuevos espacios. EN: Actas (2017). Ensenada : Universidad Nacional de La Plata. Facultad de Humanidades y Ciencias de la Educación. Departamento de Educación Física. En Memoria Académica. Disponible en: http://www.memoria.fahce.unlp.edu.ar/trab_event os/ev.10205/ev.10205.pdf

Timarán Benjumea, A., Umbarila Quintana, C. (2016). La preparación Fisica integrada para la pre-temporada en el futbol. Disponible en: http://bibliotecadigital.univalle.edu.co/bitstream/10893/9391/1/3484-0510919.pdf

Van der Horst, N.,Wouter Smits, D.,Petersen, J., Goedhart, E. \& Backx, F.(2015). The Preventive Effect of the Nordic Hamstring Exercise on Hamstring Injuries in Amateur Soccer Players. Recuperado de: https://www.researchgate.net/publication/260718483_The_Preventive_Effect_of_the_Nordic_Hamstri ng Exercise on Hamstring Injuries in Amateur Soccer Players A Randomized Controlled Trial

Vera Rivera, J. \& Merchán Osorio, R. (2011). La planificación contemporánea en el entrenamiento del fútbol. EFDeportes.com, Revista Digital, 155. Recuperado de: http://www.efdeportes.com/efd155/la-planificacion-en -el-entrenamiento-del-futbol.htm

Yanci, J. y Los Arcos, A. (2015). Relación entre distintos test de campo de condición física en jugadores de fútbol amateur. Revista Iberoamericana de Ciencias de la Actividad Fisica y el Deporte, 4(1), 42-52. Recuperado de: ht tps://www.researchgate.net/publication/275970097 